\title{
Cooperative order and excitation spectra in the bicomponent spin networks
}

\author{
Bao Xu, Han-Ting Wang, and Yupeng Wang \\ Beijing National Laboratory for Condensed Matter Physics, \\ Institute of Physics, Chinese Academy of Sciences, Beijing 100080, China
}

\begin{abstract}
A ferrimagnetic spin model composed of $S=\frac{1}{2}$ spin-dimers and $S=\frac{5}{2}$ spin-chains is studied by combining the bond-operator representation (for $S=\frac{1}{2}$ spin-dimers) and Holstein-Primakoff transformation (for $S=\frac{5}{2}$ spins). A finite interaction $J_{\mathrm{DF}}$ between the spin-dimer and the spin chain makes the spin chains ordered antiferromagnetically and the spin dimers polarized. The effective interaction between the spin chains, mediated by the spin dimers, is calculated up to the third order. The staggered magnetization in the spin dimer is shown proportional to $J_{\mathrm{DF}}$. It presents an effective staggered field reacting on the spin chains. The degeneracy of the triplons is lifted due to the chain magnetization and a mode with longitudinal polarization is identified. Due to the triplon-magnon interaction, the hybridized triplon-like excitations show different behaviors near the vanishing $J_{\mathrm{DF}}$. On the other hand, the hybridized magnon-like excitations open a gap $\Delta_{A} \sim J_{\mathrm{DF}}$. These results consist well with the experiments on $\mathrm{Cu}_{2} \mathrm{Fe}_{2} \mathrm{Ge}_{4} \mathrm{O}_{13}$.
\end{abstract}

PACS numbers: 75.10.Jm, 75.50.-y, 05.30.Jp

\section{INTRODUCTION}

Quantum magnetism has received considerable attention from both theoretical and experimental points of view in the past decades. Some low-dimensional magnets, for example, antiferromagnetic spin chains with half odd integer spins are gapless and have a disordered ground state; while some others, such as antiferromagnetic spin chains with integer spins, spin ladders and dimerized spin chains, are gapped and disordered. In two dimensional cases, Heisenberg antiferromagnets in the square lattice are gapless and have ordered ground states at zero temperature. Besides the dimensional effect, various frustration and anisotropy cause novel and complex phenomena. Recently, bicomponent systems combining two different spin frameworks have been realized experimentally in $\mathrm{R}_{2} \mathrm{BaNiO}_{5}{ }^{1,2}$, $\mathrm{Cu}_{2} \mathrm{Fe}_{2} \mathrm{Ge}_{4} \mathrm{O}_{13}{ }_{3,4,5,6}, \mathrm{Cu}_{2} \mathrm{CdB}_{2} \mathrm{O}_{6}{ }^{7}$ and $\mathrm{Cu}_{3} \mathrm{Mo}_{2} \mathrm{O}_{9}{ }^{8}$. Among them, $\mathrm{Cu}_{2} \mathrm{Fe}_{2} \mathrm{Ge}_{4} \mathrm{O}_{13}$, which incorporates intercalated $\mathrm{Cu}^{2+}$ spin dimers $\left(S=\frac{1}{2}\right)$ and $\mathrm{Fe}^{3+}$ spin chains $\left(S=\frac{5}{2}\right)$, is most extensively studied. Below $T_{N}=39 \mathrm{~K}$, a cooperative order was observed by the measurements of susceptibility and heat capacity $\stackrel{4}{*}$. At $T=1.5 \mathrm{~K}$, the estimated $m_{\mathrm{Cu}}=0.38$ $\mu_{B}$ and $m_{\mathrm{Fe}}=3.62 \mu_{B}$. Compared to the classical expectation value of $1 \mu_{B}, m_{\mathrm{Cu}}$ is drastically suppressed and keeps proportional to $m_{\mathrm{Fe}}$ at all temperatures. By detailed inelastic neutron scattering study $\underline{\underline{4}, 5,6}, \mathrm{Cu}_{2} \mathrm{Fe}_{2} \mathrm{Ge}_{4} \mathrm{O}_{13}$ was found to exhibit two types of spin excitations with separate energy scales. Although the $\mathrm{Fe}^{3+}$-centered low-energy spin excitations can be well interpreted by the spin wave theory and a small gap of about $1 \mathrm{meV}$ is estimated, the $\mathrm{Cu}^{2+}$ centered high-energy part is less understood. Masuda et. a ${ }^{\underline{4}}$ guessed the presence of a triplet mode, which should have longitudinal polarization and be totally incompatible with conventional spin wave theory. In this paper, we combine bond operator representation ${ }^{9}$ and Holstein-Primakoff transformation 10 to study this bicomponent system.

Since the exchange coupling along the c-axis is pretty weak, the real geometry of $\mathrm{Cu}_{2} \mathrm{Fe}_{2} \mathrm{Ge}_{4} \mathrm{O}_{13}$ could be simplified as a two-dimensional topologically equivalent mode $\underline{l}^{3}$ (Fig, (1). The model Hamiltonian consists of the $\mathrm{Cu}-\mathrm{Cu}$, the $\mathrm{Fe}-\mathrm{Fe}$ and the $\mathrm{Cu}-\mathrm{Fe}$ interactions:

$$
H=H_{\mathrm{Cu}}+H_{\mathrm{Fe}}+H_{\mathrm{Cu}-\mathrm{Fe}},
$$

with

$$
\begin{aligned}
H_{\mathrm{Cu}}= & \sum_{\vec{r}} J_{\mathrm{D}}\left(\hat{T}_{\vec{r}, 1} \cdot \hat{T}_{\vec{r}, 2}+\hat{T}_{\vec{r}, 3} \cdot \hat{T}_{\vec{r}, 4}\right), \\
H_{\mathrm{Fe}}= & \sum_{\vec{r}} \frac{1}{2} J_{\mathrm{a}}\left(\hat{S}_{\vec{r}, 1} \cdot \hat{S}_{\vec{r}+\vec{a}, 2}+\hat{S}_{\vec{r}+\vec{a}, 3} \cdot \hat{S}_{\vec{r}, 4}\right. \\
& \left.+\hat{S}_{\vec{r}-\vec{a}, 1} \cdot \hat{S}_{\vec{r}, 2}+\hat{S}_{\vec{r}, 3} \cdot \hat{S}_{\vec{r}-\vec{a}, 4}\right) \\
& +J_{\mathrm{b}}\left(\hat{S}_{\vec{r}, 3} \cdot \hat{S}_{\vec{r}, 2}+\frac{1}{2} \hat{S}_{\vec{r}, 1} \cdot \hat{S}_{\vec{r}-\vec{b}, 4}+\frac{1}{2} \hat{S}_{\vec{r}+\vec{b}, 1} \cdot \hat{S}_{\vec{r}, 4}\right), \\
H_{\mathrm{Cu}-\mathrm{Fe}}= & \sum_{\vec{r}, i=1,2,3,4} J_{\mathrm{DF}} \hat{T}_{\vec{r}, i} \cdot \hat{S}_{\vec{r}, i},
\end{aligned}
$$

where $J_{\mathrm{D}}$ and $J_{\mathrm{DF}}$ denote the $\mathrm{Cu}-\mathrm{Cu}$ and $\mathrm{Cu}-\mathrm{Fe}$ interactions respectively, and $J_{\mathrm{a}(\mathrm{b})}$ the Fe-Fe exchange constant along $\mathrm{a}(\mathrm{b})$ direction. By neutron inelastic scattering and neutron diffraction $\underset{\underline{6}}{ }, J_{\mathrm{D}}$ was found dominantly larger than other 


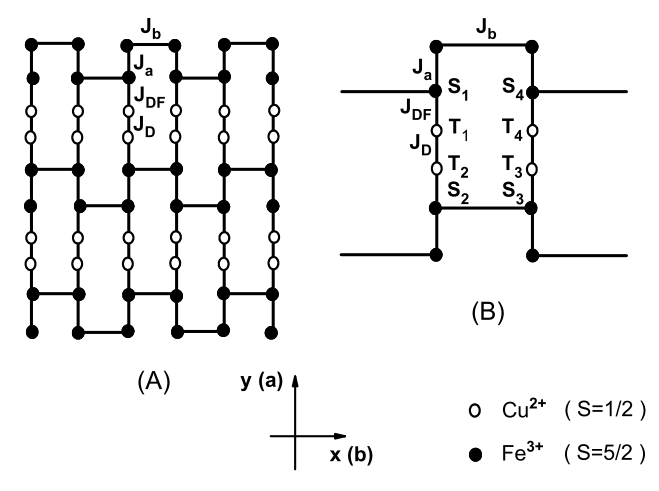

FIG. 1: The geometry of the topologically equivalent spin lattice of $\mathrm{Cu}_{2} \mathrm{Fe}_{2} \mathrm{Ge}_{4} \mathrm{O}_{13}$. The $\mathrm{Cu}-\mathrm{Cu}$ interaction $J_{\mathrm{D}}$ is dominantly larger than the Fe-Fe interaction $J_{\mathrm{a}(\mathrm{b})}$ and the $\mathrm{Cu}-\mathrm{Fe}$ interaction $J_{\mathrm{DF}}$. The weak interchain interaction along c-direction is neglected for simplicity.

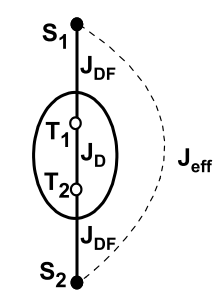

FIG. 2: The effective four-spin model.

interactions and $J_{\mathrm{b}}$ is very close to $J_{\mathrm{a}}$. The $\mathrm{Fe}^{3+}$ has $\operatorname{spin} S=\frac{5}{2}$ and the $\mathrm{Cu}^{2+}$ has $\operatorname{spin} T=\frac{1}{2}$. Single-ion anisotropy and external magnetic field are not considered here. With a vanishing $J_{\mathrm{DF}}$, the model reduces to the independent one-dimensional Heisenberg antiferromagnetic chains with $S=\frac{5}{2}$ and isolated spin dimers with $T=\frac{1}{2}$. With a finite $J_{\mathrm{DF}}$, an effective interaction between the $\mathrm{Fe}^{3+}$ chains, mediated by the $\mathrm{Cu}^{2+}$ dimers, will make the $\mathrm{Fe}^{3+}$ spins stay in the Néel state. (The small Fe-Fe interactions along c-direction reinforce this order). On the other hand, the local magnetization of $\mathrm{Fe}^{3+}$ spins will tend to excite the $\mathrm{Cu}^{2+}$ dimers from the singlet to the triplet and the $\mathrm{Cu}^{2+}$ spins will then show local magnetizations. In the following, we study the effective interaction between the $\mathrm{Fe}^{3+}$ chains $^{3}$ mediated by the $\mathrm{Cu}^{2+}$ dimers perturbatively and the $\mathrm{Cu}^{2+}$ magnetization induced by the local $\mathrm{Fe}^{3+}$ spins(section II). In section III, we study the excitation spectra of the mixed spin system. A summary is given in section IV.

\section{INDIRECT FE-FE MAGNETIC INTERACTION AND CU ${ }^{2+}$ MAGNETIZATION POLARIZED BY THE FE $^{3+}$ SPIN}

As mentioned before, $J_{\mathrm{D}}$ is much larger than $J_{\mathrm{a}(\mathrm{b})}$ and $J_{\mathrm{DF}}$. Considering the low energy excitations only and integrating out the degrees of freedom of the spin dimers, we could obtain an effective Fe-Fe exchange $J_{\text {eff }}$ mediated by isolated spin- $\frac{1}{2}$ dimers $\frac{11}{}$. In the model Hamiltonian(eq. (1)), $H_{\mathrm{Cu}-\mathrm{Fe}}$ could be treated as a small amount and $H_{\mathrm{Fe}}$ will be neglected for the moment since no $\mathrm{Cu}^{2+}$ spin operators are involved in it. As shown in Fig. 2, the model then reduces to a four-spin system

$$
\mathcal{H}=H_{0}+H^{\prime}
$$

with $H_{0}=J_{\mathrm{D}} \hat{T}_{1} \cdot \hat{T}_{2}$ and $H^{\prime}=J_{\mathrm{DF}}\left(\hat{T}_{1} \cdot \hat{S}_{1}+\hat{T}_{2} \cdot \hat{S}_{2}\right)$. 
$H_{0}$ has a singlet ground state $\mid s>$ with a ground state energy $E_{0}=-\frac{3}{4} J_{\mathrm{D}}$. Its excited states are triplets $\mid t_{\alpha}>$ with $\alpha=0, \pm 1$, corresponding to the total $z$ component $T_{1 z}+T_{2 z}=0, \pm 1$. The eigenenergy of the excited states is $E_{1, \alpha}=\frac{1}{4} J_{\mathrm{D}}$. The first order perturbation of $H_{\mathrm{Cu}-\mathrm{Fe}}$ is $\left.\mathcal{H}_{1}=<s\left|H_{\mathrm{Cu}-\mathrm{Fe}}\right| s\right\rangle=0$. The 2ndorder perturbation $\mathcal{H}_{2}=\sum_{\alpha} \frac{\left\langle s\left|H_{\mathrm{Cu}-\mathrm{Fe}}\right| t_{\alpha}><t_{\alpha}\left|H_{\mathrm{Cu}-\mathrm{Fe}}\right| s\right\rangle}{E_{0}-E_{1 \alpha}}=\frac{J_{\mathrm{DF}}^{2}}{2 J_{\mathrm{D}}} \hat{S}_{1} \cdot \hat{S}_{2}$. The 3rd-order perturbation is $\mathcal{H}_{3}=$ $\sum_{\alpha, \beta} \frac{\left\langle s\left|H_{\mathrm{Cu}-\mathrm{Fe}}\right| t_{\alpha}><t_{\alpha}\left|H_{\mathrm{Cu}-\mathrm{Fe}}\right| t_{\beta}><t_{\beta}\left|H_{\mathrm{Cu}-\mathrm{Fe}}\right| s\right\rangle}{\left(E_{0}-E_{1, \alpha}\right)\left(E_{0}-E_{1, \beta}\right)}=\frac{3 J_{\mathrm{DF}}^{3}}{4 J_{\mathrm{D}}^{2}} \hat{S}_{1} \cdot \hat{S}_{2}$. Thus, up to the third order, the effective exchange coupling mediated by the spin- $1 / 2$ dimer is obtained as

$$
\mathcal{H}_{\mathrm{eff}}=J_{\mathrm{eff}} \hat{S}_{1} \cdot \hat{S}_{2}
$$

with $J_{\mathrm{eff}}=\frac{J_{\mathrm{DF}}^{2}}{2 J_{\mathrm{D}}}\left(1+\frac{3 J_{\mathrm{DF}}}{2 J_{\mathrm{D}}}\right)$.

With the effective interaction between the $\mathrm{Fe}^{3+}$ spin chains considered, the $\mathrm{Fe}^{3+}$ spins will order antiferromagnetically. We use a molecular field approximation to study the effects of the staggered $\mathrm{Fe}^{3+}$ magnetization on the $\mathrm{Cu}^{2+}$ spin dimers. It is relevant at a higher energy range (comparable to the dimer gap), where the dynamics of the system is dominated by the dimers and the chain freedom can be effectively integrated out. The four spin Hamiltonian becomes

$$
\mathcal{H}=J_{\mathrm{D}} \hat{T}_{1} \cdot \hat{T}_{2}+J_{\mathrm{DF}} m_{\mathrm{Fe}}\left(T_{1 z}-T_{2 z}\right),
$$

where $m_{\mathrm{Fe}}=<S_{z}>$ is the staggered magnetization of $\mathrm{Fe}^{3+}$ spin. This approximate Hamiltonian can be exactly diagonalized with the bases $\mid s>$ and $\mid t_{\alpha}>(\alpha=0, \pm 1)$. The eigenvalues are $e_{0}=-\frac{1}{4} J_{\mathrm{D}}-\frac{1}{2} J_{\mathrm{D}} \sqrt{1+\frac{4 J_{\mathrm{DF}}^{2} m_{\mathrm{Fe}}^{2}}{J_{\mathrm{D}}^{2}}}$, $e_{1}=-\frac{1}{4} J_{\mathrm{D}}+\frac{1}{2} J_{\mathrm{D}} \sqrt{1+\frac{4 J_{\mathrm{DF}}^{2} m_{\mathrm{Fe}}^{2}}{J_{\mathrm{D}}^{2}}}$, and $e_{2}=e_{3}=\frac{1}{4} J_{\mathrm{D}}$. The corresponding eigenstates are $\left|\psi_{i}>=a_{i}\right| s>+b_{i} \mid t_{0}>$ $(i=0,1)$ with $a_{i}=\frac{J_{\mathrm{DF}} m_{\mathrm{Fe}}}{\sqrt{\left(e_{i}+\frac{3}{4} J_{\mathrm{D}}\right)^{2}+J_{\mathrm{DF}}^{2} m_{\mathrm{Fe}}^{2}}}$ and $b_{i}=\frac{e_{i}+\frac{3}{4} J_{\mathrm{D}}}{\sqrt{\left(e_{i}+\frac{3}{4} J_{\mathrm{D}}\right)^{2}+J_{\mathrm{DF}}^{2} m_{\mathrm{Fe}}^{2}}},\left|\psi_{3}>=\right| t_{1}>$ and $\left|\psi_{4}>=\right| t_{-1}>$. From the ground state $\left|\psi_{0}\right\rangle$, we find that $\left|t_{0}\right\rangle$ is partly excited and the $\mathrm{Cu}^{2+}$ spins now get a finite staggered magnetization: $m_{\mathrm{Cu}}=<T_{2 z}>=-<T_{1 z}>=<\psi_{0}\left|T_{2 z}\right| \psi_{0}>=a_{0} b_{0}$. When $m_{\mathrm{Fe}}$ or $\frac{J_{\mathrm{DF}}}{J_{\mathrm{D}}}$ is small, we have $m_{\mathrm{Cu}} \approx \frac{J_{\mathrm{DF}}}{J_{\mathrm{D}}} m_{\mathrm{Fe}}$, which agree well with the experiment $\stackrel{4}{ }$.

\section{EXCITATIONS IN THE BICOMPONENT SYSTEM}

In the $\mathrm{Fe}-\mathrm{Cu}$ spin system, if we neglect the interaction between the $\mathrm{Cu}^{2+}$ and $\mathrm{Fe}^{3+}$ spins, the $\mathrm{Fe}^{3+}$ spins will be in the Néel state (considering the antiferromagnetic interaction along c-direction) and its excitations are spin-waves (magnons); while the $\mathrm{Cu}^{2+}$ dimers are in the singlet states and the excitations are triplets (triplons). When the $\mathrm{Cu}-\mathrm{Fe}$ interactions are switched on, the Néel order in the $\mathrm{Fe}^{3+}$ spin network is reinforced and a staggered magnetization in the $\mathrm{Cu}^{2+}$ spins are induced. The magnon and triplon interact with each other and the hybridized excitations show interesting behavior. In the following, we study these excitations.

For $\mathrm{Cu}^{2+}$ spin dimers, we use the bond operator representation. With the definition of $\left|s>=s^{\dagger}\right| 0>,\left|t_{0}>=t^{\dagger}\right| 0>$, $\left|t_{-1}>=d^{\dagger}\right| 0>,\left|t_{1}>=u^{\dagger}\right| 0>$ and $s^{\dagger} s+t^{\dagger} t+d^{\dagger} d+u^{\dagger} u=1$, the $\mathrm{Cu}^{2+}$ spin operators are expressed as $\mathrm{s}^{\mathrm{9}}$ :

$$
\begin{aligned}
T_{\sigma}^{+} & =\frac{1}{\sqrt{2}}\left[-\left(u_{\sigma}^{\dagger} s_{\sigma}-s_{\sigma}^{\dagger} d_{\sigma}\right)+t_{\sigma}^{\dagger} d_{\sigma}+u_{\sigma}^{\dagger} t_{\sigma}\right] ; \\
T_{\sigma+1}^{+} & =\frac{1}{\sqrt{2}}\left[\left(u_{\sigma}^{\dagger} s_{\sigma}-s_{\sigma}^{\dagger} d_{\sigma}\right)+t_{\sigma}^{\dagger} d_{\sigma}+u_{\sigma}^{\dagger} t_{\sigma}\right] ; \\
T_{\sigma}^{z} & =\frac{1}{2}\left[\left(s_{\sigma}^{\dagger} t_{\sigma}+t_{\sigma}^{\dagger} s_{\sigma}\right)+u_{\sigma}^{\dagger} u_{\sigma}-d_{\sigma}^{\dagger} d_{\sigma}\right] ; \\
T_{\sigma+1}^{z} & =\frac{1}{2}\left[-\left(s_{\sigma}^{\dagger} t_{\sigma}+t_{\sigma}^{\dagger} s_{\sigma}\right)+u_{\sigma}^{\dagger} u_{\sigma}-d_{\sigma}^{\dagger} d_{\sigma}\right] ;
\end{aligned}
$$

where, $\sigma=1,3$, corresponding to the pairs of $\left(\hat{T}_{1}, \hat{T}_{2}\right)$ and $\left(\hat{T}_{3}, \hat{T}_{4}\right)$, respectively.

We apply Holstein-Primakoff transformation to $\mathrm{Fe}^{3+}$ spins ${ }^{10}$. Supposing $\hat{S}_{1}$ and $\hat{S}_{3}$ point up and $\hat{S}_{2}$ and $\hat{S}_{4}$ point down, we have

$$
\begin{aligned}
S_{i}^{+} & =\sqrt{2 S-a_{i}^{\dagger} a_{i}} a_{i}, \\
S_{i}^{-} & =a_{i}^{\dagger} \sqrt{2 S-a_{i}^{\dagger} a_{i}}, \\
S_{i}^{z} & =S-a_{i}^{\dagger} a_{i}, \quad i=1,3 ;
\end{aligned}
$$


and

$$
\begin{aligned}
S_{j}^{+} & =b_{j}^{\dagger} \sqrt{2 S-b_{j}^{\dagger} b_{j}}, \\
S_{j}^{-} & =\sqrt{2 S-b_{j}^{\dagger} b_{j}} b_{j}, \\
S_{j}^{z} & =b_{j}^{\dagger} b_{j}-S, \quad j=2,4 .
\end{aligned}
$$

Before we substitute these representations into Hamiltonian (1), some approximations have to be made. As found in section II, the $\mathrm{Cu}^{2+}$ dimers are in a mixed state of $|s\rangle$ and $\left|t_{0}\right\rangle$. We introduce another four operators as ${ }^{12,13}$

$$
\left(\begin{array}{c}
X_{\sigma} \\
Y_{\sigma}
\end{array}\right)=\left(\begin{array}{cc}
\cos \theta & -\sin \theta \\
\sin \theta & \cos \theta
\end{array}\right)\left(\begin{array}{c}
s_{\sigma} \\
t_{\sigma}
\end{array}\right)
$$

and

$$
\left(\begin{array}{c}
s_{\sigma} \\
t_{\sigma}
\end{array}\right)=\left(\begin{array}{cc}
\cos \theta & \sin \theta \\
-\sin \theta & \cos \theta
\end{array}\right)\left(\begin{array}{c}
X_{\sigma} \\
Y_{\sigma}
\end{array}\right)
$$

with $\sigma=1,3$ and suppose $X_{\sigma}$ bosons are condensed with $\left.\left\langle X_{\sigma}\right\rangle=<X_{\sigma}^{\dagger}\right\rangle=1$, which means a long range order in the $\mathrm{Cu}^{2+}$ spin network. $\theta$ will be determined variationally or by canceling the single-operator terms.

For the Holstein-Primakoff transformation, we employ the usual linear approximation: $S_{i}^{+}=\sqrt{2 S} a_{i}, S_{i}^{-}=\sqrt{2 S} a_{i}^{\dagger}$, $S_{i}^{z}=S-a_{i}^{\dagger} a_{i}, i=1,3$ and $S_{j}^{+}=\sqrt{2 S} b_{j}^{\dagger}, S_{j}^{-}=\sqrt{2 S} b_{j}, S_{j}^{z}=-S+b_{j}^{\dagger} b_{j}, j=2,4$. Substituting these transformations into Hamiltonian (1), we get

$$
\begin{aligned}
H= & \sum_{\vec{r}}\left[\left(J_{\mathrm{DF}} S \cos 2 \theta-\frac{1}{2} J_{\mathrm{D}} \sin 2 \theta\right)\left(Y_{1 \vec{r}}^{\dagger}+Y_{1 \vec{r}}+Y_{3 \vec{r}}^{\dagger}+Y_{3 \vec{r}}\right)+\left(J_{\mathrm{DF}} S \sin 2 \theta+J_{\mathrm{D}} \cos 2 \theta\right)\left(Y_{1 \vec{r}}^{\dagger} Y_{1 \vec{r}}+Y_{3 \vec{r}}^{\dagger} Y_{3 \vec{r}}\right)\right. \\
& +\frac{1}{2} J_{\mathrm{D}}(1+\cos 2 \theta)\left(u_{1 \vec{r}}^{\dagger} u_{1 \vec{r}}+d_{1 \vec{r}}^{\dagger} d_{1 \vec{r}}+u_{3 \vec{r}}^{\dagger} u_{3 \vec{r}}+d_{3 \vec{r}}^{\dagger} d_{3 \vec{r}}\right)+\left(2 J_{\mathrm{a}} S+\frac{1}{2} J_{\mathrm{DF}} \sin 2 \theta\right)\left(a_{1 \vec{r}}^{\dagger} a_{1 \vec{r}}+b_{2 \vec{r}}^{\dagger} b_{2 \vec{r}}+a_{3 \vec{r}}^{\dagger} a_{3 \vec{r}}+b_{4 \vec{r}}^{\dagger} b_{4 \vec{r}}\right) \\
& -\frac{\sqrt{2 S}}{2} J_{\mathrm{DF}} \sin \left(\theta+\frac{\pi}{4}\right)\left(a_{1 \vec{r}}^{\dagger} u_{1 \vec{r}}^{\dagger}+a_{3 \vec{r}}^{\dagger} u_{3 \vec{r}}^{\dagger}+b_{2 \vec{r}} d_{1 \vec{r}}+b_{4 \vec{r}} d_{3 \vec{r}}+h . c .\right) \\
& -\frac{\sqrt{2 S}}{2} J_{\mathrm{DF}} \sin \left(\theta-\frac{\pi}{4}\right)\left(a_{1 \vec{r}}^{\dagger} d_{1 \vec{r}}+a_{3 \vec{r}}^{\dagger} d_{3 \vec{r}}+u_{1 \vec{r}}^{\dagger} b_{2 \vec{r}}+u_{3 \vec{r}}^{\dagger} b_{4 \vec{r}}+h . c .\right) \\
& \left.+J_{\mathrm{a}} S\left(a_{3 \vec{r}} b_{2 \vec{r}}+h . c .\right)+\frac{1}{2} J_{\mathrm{a}} S\left(a_{1 \vec{r}} b_{4 \vec{r}-\vec{b}}+a_{1 \vec{r}+\vec{b}}^{\dagger} b_{4 \vec{r}}^{\dagger}+h . c .\right)+\frac{1}{2} J_{\mathrm{a}} S\left(a_{1 \vec{r}} b_{2 \vec{r}+\vec{a}}+a_{3 \vec{r}} b_{4 \vec{r}-\vec{a}}+a_{1 \vec{r}-\vec{a}}^{\dagger} b_{2 \vec{r}}^{\dagger}+a_{3 \vec{r}+\vec{a}}^{\dagger} b_{4 \vec{r}}^{\dagger}+h . c .\right)\right] \\
& -2 N J_{\mathrm{DF}} S \sin 2 \theta-N\left(\frac{1}{2}+\cos 2 \theta\right) J_{\mathrm{D}}-4 N J_{\mathrm{a}} S^{2}+3-\text { operator terms }+4-\text { operator terms. }
\end{aligned}
$$

The 3-operator and 4-operator terms are omitted here for simplicity. By letting the coefficient of the single-operator terms be zero, we get

$$
\theta=\frac{1}{2} \arctan \frac{2 J_{\mathrm{DF}} S}{J_{\mathrm{D}}}
$$

Correspondingly, at zero temperature, we get the staggered magnetization at the $\mathrm{Cu}$ site as

$$
m_{\mathrm{Cu}}=\left|<T_{1 z}>\right|=\frac{1}{2} \sin 2 \theta=\frac{\frac{J_{\mathrm{DF}}}{J_{\mathrm{D}}} S}{\sqrt{1+\left(2 \frac{J_{\mathrm{DF}}}{J_{\mathrm{D}}}\right)^{2}}} .
$$

When $J_{\mathrm{DF}}<<J_{\mathrm{D}}, \theta \approx \frac{J_{\mathrm{DF}} S}{J_{\mathrm{D}}}$ and $m_{\mathrm{Cu}} \approx \frac{J_{\mathrm{DF}} S}{J_{\mathrm{D}}}$. This result is consistent with $m_{\mathrm{Cu}} \approx \frac{J_{\mathrm{DF}}}{J_{\mathrm{D}}} m_{\mathrm{Fe}}$, which we get from the molecular field approximation in section II.

After Fourier transformation, the Hamiltonian can be written as

$$
H=\sum_{k} \omega_{k}^{Y}\left(Y_{1, k}^{\dagger} Y_{1, k}+Y_{3, k}^{\dagger} Y_{3, k}\right)+\Psi_{k}^{\dagger} H_{k} \Psi_{k}+C,
$$

where,

$$
\begin{aligned}
\omega_{k}^{Y} & =J_{\mathrm{DF}} S \sin 2 \theta+J_{\mathrm{D}} \cos 2 \theta \\
C & =-2 N J_{\mathrm{DF}} S \sin 2 \theta-N\left(\frac{1}{2}+\cos 2 \theta\right) J_{\mathrm{D}}-4 N J_{\mathrm{a}} S^{2}, \\
\Psi_{k}^{\dagger} & =\left(a_{1, k}^{\dagger}, b_{2,-k}, a_{3, k}^{\dagger}, b_{4,-k}, d_{1, k}^{\dagger}, u_{1,-k}, d_{3, k}^{\dagger}, u_{3,-k}\right),
\end{aligned}
$$




$$
H_{k}=\left(\begin{array}{cccccccc}
a & \delta e^{-i k_{y}} & 0 & \delta e^{i k_{x}} & -\gamma \cos \nu & \gamma \sin \nu & 0 & 0 \\
\delta e^{i k_{y}} & a & \delta & 0 & \gamma \sin \nu & -\gamma \cos \nu & 0 & 0 \\
0 & \delta & a & \delta e^{i k_{y}} & 0 & 0 & -\gamma \cos \nu & \gamma \sin \nu \\
\delta e^{-i k_{x}} & 0 & \delta e^{-i k_{y}} & a & 0 & 0 & \gamma \sin \nu & -\gamma \cos \nu \\
-\gamma \cos \nu & \gamma \sin \nu & 0 & 0 & d & 0 & 0 & 0 \\
\gamma \sin \nu & -\gamma \cos \nu & 0 & 0 & 0 & d & 0 & 0 \\
0 & 0 & -\gamma \cos \nu & \gamma \sin \nu & 0 & 0 & d & 0 \\
0 & 0 & \gamma \sin \nu & -\gamma \cos \nu & 0 & 0 & 0 & d
\end{array}\right)
$$

with

$$
\begin{aligned}
a & =2 J_{\mathrm{a}} S+\frac{1}{2} J_{\mathrm{DF}} \sin 2 \theta, \\
d & =\frac{1}{2} J_{\mathrm{D}}(1+\cos 2 \theta), \\
\gamma & =-\sqrt{\frac{S}{2}} J_{\mathrm{DF}}, \\
\delta & =J_{\mathrm{a}} S \\
\nu & =\theta+\frac{\pi}{4} .
\end{aligned}
$$

Compared with the conventional spin wave theory, the magnon gets an additional energy of $\frac{1}{2} J_{\mathrm{DF}} \sin 2 \theta=J_{\mathrm{DF}} m_{\mathrm{Cu}}$, which comes from the polarization of the $\mathrm{Cu}^{2+}$ spin dimer. As a result, the magnon excitations show a tiny gap. We will discuss it later. The Hamiltonian can be diagonalized as

$$
H=\sum_{k} \sum_{i=1,8} \omega_{i k} \alpha_{i k}^{\dagger} \alpha_{i k}+\sum_{k} \omega_{k}^{Y}\left(Y_{1 k}^{\dagger} Y_{1 k}+Y_{3 k}^{\dagger} Y_{3 k}\right)
$$

where, a constant has been neglected and the expressions for $\omega_{i k}$ are given in Appendix A.

The triplon excitations $Y_{\sigma k}(\sigma=1,3)$ do not interact with the magnons. They are dispersionless. The spectrum $\omega_{k}^{Y}=J_{\mathrm{D}}\left[1+2\left(\frac{J_{\mathrm{DF}} S}{J_{\mathrm{D}}}\right)^{2}\right] / \sqrt{1+4\left(\frac{J_{\mathrm{DF}} S}{J_{\mathrm{D}}}\right)^{2}} \approx J_{\mathrm{D}}\left[1+2\left(\frac{J_{\mathrm{DF}} S}{J_{\mathrm{D}}}\right)^{4}\right]$. It increases with increasing $J_{\mathrm{DF}}$ and returns to the singlet-triplet gap when $J_{\mathrm{DF}}=0$. With the $\mathrm{Cu}^{2+}$ spin operator (eq. (6)) expressed by the $X_{\sigma}$ and $Y_{\sigma}$ (eq. (10)) and making mean-field approximation of $\left\langle X_{\sigma}\right\rangle=1$, we find $T^{z}$ proportional to $Y$ and $Y^{\dagger}$ and $T^{ \pm}\left(T^{x(y)}\right)$ proportional to $u\left(u^{\dagger}\right)$ and $d\left(d^{\dagger}\right)$. Thus the operators $Y_{\sigma k}=\sin \theta s_{\sigma k}+\cos \theta t_{\sigma k}(\sigma=1,3)$ describe the longitudinal fluctuations and we believe they are the modes having longitudinal polarization and totally incompatible with conventional spin wave theory, as guessed by Masuda et. $\mathrm{al}^{\underline{4}}$. It is emphasized that these modes cannot be obtained by spin wave theory or effective spin wave theory $\underline{\underline{6}}$.

Among the eight $\alpha_{i k}$ excitations, four branches are magnon-like and another four are triplon-like. We denote them by $A_{1 k}, A_{3 k}, B_{2 k}, B_{4 k}$ and $D_{1 k}, D_{3 k}, U_{1 k}, U_{3 k}$ respectively. The corresponding spectra are represented by $\omega_{k}^{A_{1}}, \omega_{k}^{A_{3}}, \omega_{k}^{B_{2}}, \omega_{k}^{B_{4}}$ and $\omega_{k}^{D_{1}}, \omega_{k}^{D_{3}}, \omega_{k}^{U_{1}}, \omega_{k}^{U_{3}}$. They are two-fold degenerate. At $J_{\mathrm{DF}}=0$, they return to the pure magnon spectra $\omega_{k}^{A / B}=J_{\mathrm{a}} S \sqrt{2\left(1 \mp \cos \frac{k_{x}}{2}\right)}$ and pure triplon spectra $\omega_{k}^{U / D}=J_{\mathrm{D}}$. The magnons are gapless and the triplons are dispersionless. At a finite $J_{\mathrm{DF}}$, the magnons become gapful and the triplons become mobile due to the magnon-triplon interaction.

Experimentally, $J_{\mathrm{D}}$ and $J_{\mathrm{a}}$ were determined as $J_{\mathrm{D}}=24 \mathrm{meV}$ and $J_{\mathrm{a}}=1.6 \mathrm{meV}$. The reported $J_{\mathrm{DF}}$ is discrepant such as $2.4 \mathrm{meV}, 0.9 \mathrm{meV}$ or $2.0 \mathrm{meV}$. In Fig. 3 and 4, we show the numerically calculated magnon-like and triplon-like excitation spectra, respectively, with $J_{\mathrm{D}}=24 \mathrm{meV}, J_{\mathrm{a}}=1.6 \mathrm{meV}$ and $J_{\mathrm{DF}}=1 \mathrm{meV}$. The calculated magnon gap is $1.27 \mathrm{meV}$. Experimentally, a small empirical anisotropy gap is estimated as $1 \mathrm{meV}$ (spin wave theory ${ }^{6}$ ) or 2.02 $\mathrm{meV}$ (effective spin wave theor $\mathrm{y}^{\underline{4}}$ ). We find that the gap largely depends on $J_{\mathrm{DF}}$. The triplon-like excitations have a dispersion with small amplitude.

Regarding $\frac{J_{\mathrm{DF}}}{J_{\mathrm{D}}}$ as a small amount, we could get analytical expressions for the spectra. The details are given in the Appendix. The magnon-like excitation has a gap $\Delta_{A}=\omega^{A_{\sigma}}(0, \pi)=\Delta_{B}=\omega^{B_{\sigma^{\prime}}}(2 \pi, \pi) \approx J_{D} \sqrt{\frac{2 \eta}{1-\eta^{2}}}|\rho|(\sigma=1,3$, $\left.\sigma^{\prime}=2,4\right)$ with $\eta=\frac{2 J_{\mathrm{J}} S}{J_{\mathrm{D}}}$ and $\rho=-\frac{J_{\mathrm{DF}}}{J_{\mathrm{D}}} \sqrt{\frac{S}{2}}$. It should be pointed out that the magnon-like spectra are proportional to $\left(\frac{J_{\mathrm{DF}}}{J_{\mathrm{D}}}\right)^{2}$ away from the momentum of $(0, \pi)$ or $(2 \pi, \pi)$ (see eq. (A9)). The gap of the triplon-like excitations is $\Delta_{U}=\omega^{U_{\sigma}}(2 \pi, \pi)=\Delta_{D}=\omega^{D_{\sigma}}(0, \pi) \approx J_{\mathrm{D}}\left(1-\frac{\eta}{1-\eta^{2}} \rho^{2}\right)(\sigma=1,3)$. When $\eta<<1$, the gaps can be further simplified 


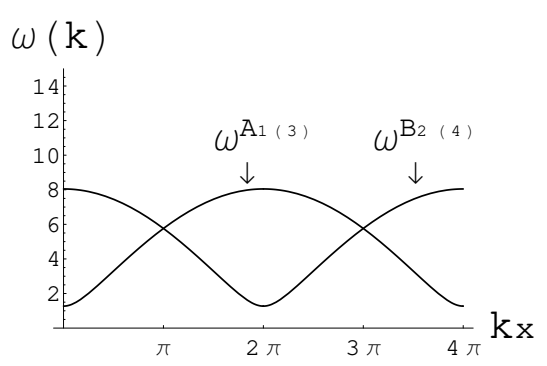

FIG. 3: Spectra of the magnon-like excitations $\omega_{k}^{A_{1}}, \omega_{k}^{A_{3}}, \omega_{k}^{B_{2}}, \omega_{k}^{B_{4}}$ with $J_{\mathrm{D}}=24 \mathrm{meV}, J_{\mathrm{a}}=1.6 \mathrm{meV}$ and $J_{\mathrm{DF}}=1 \mathrm{meV}$. Compared to the case of $J_{\mathrm{DF}}=0$, a small gap opens.

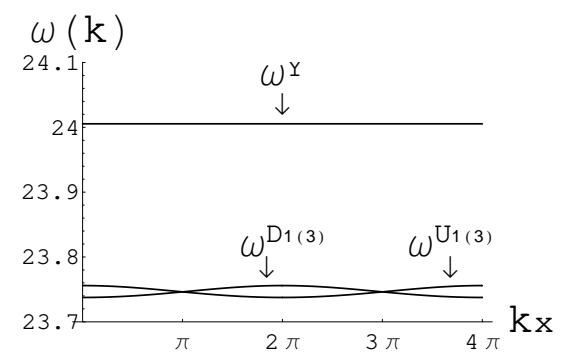

FIG. 4: Spectra of the triplon-like excitations $\omega_{k}^{Y}, \omega_{k}^{D_{\sigma}}$ and $\omega_{k}^{U_{\sigma}}(\sigma=1,3)$ with $J_{\mathrm{D}}=24 \mathrm{meV}, J_{\mathrm{a}}=1.6 \mathrm{meV}$ and $J_{\mathrm{DF}}=1$ $\mathrm{meV}$. The dispersionless $\omega_{k}^{Y}$ is longitudinally polarized, as mentioned by Masuda et. al. $\stackrel{4}{=}$ Compared to the case of $J_{\mathrm{DF}}=0$, $\omega_{k}^{D_{\sigma}}$ and $\omega_{k}^{U_{\sigma}}(\sigma=1,3)$ show weak dispersions.

as $\Delta_{A} \approx J_{\mathrm{DF}} S \sqrt{\frac{2 J_{\mathrm{a}}}{J_{\mathrm{D}}}} \approx J_{\mathrm{DF}}$ and $\Delta_{U} \approx J_{\mathrm{D}}\left[1-\frac{J_{\mathrm{a}}}{J_{\mathrm{D}}}\left(\frac{J_{\mathrm{DF}} S}{J_{\mathrm{D}}}\right)^{2}\right] \propto\left(\frac{J_{\mathrm{DF}}}{J_{\mathrm{D}}}\right)^{2}$. These relations are numerically shown in Fig. 5. Reminding $\Delta_{Y} \propto\left(\frac{J_{\mathrm{DF}} S}{J_{\mathrm{D}}}\right)^{4}$, we find that different branches have different dependences on $\frac{J_{\mathrm{DF}}}{J_{\mathrm{D}}}$. The band width of the triplon-like excitations is $W_{U}=W_{D} \approx J_{\mathrm{D}} \frac{\eta}{1-\eta^{2}} \rho^{2} \approx J_{\mathrm{a}}\left(\frac{J_{\mathrm{DF}} S}{J_{\mathrm{D}}}\right)^{2}$. As a counterpart, an effective interaction between the $\mathrm{Cu}^{2+}$ dimers, mediated by the $\mathrm{Fe}^{3+}$ chains, can be estimated as $J_{\text {eff }}^{\prime} \sim J_{\mathrm{a}}\left(\frac{J_{\mathrm{DF}} S}{J_{\mathrm{D}}}\right)^{2}$.

Experimentally ${ }_{1}^{4}, 6$, the magnetic moment in $\mathrm{Cu}_{2} \mathrm{Fe}_{2} \mathrm{Ge}_{4} \mathrm{O}_{13}$ was found to be nearly confined in the a-c plane. Dzyaloshinskii-Moriya (DM) interaction and other insignificant anisotropy effects may exist. For simplicity, we neglect these anisotropy effects and the weak interaction along c-direction in our mean-field theory. Some remarks on the Goldstone theorem have to be made here. We start from an isotropic spin Hamiltonian and a gapless mode is expected in the ordered state because of the rotational symmetry. The small but finite magnon gap seems unwelcome. We

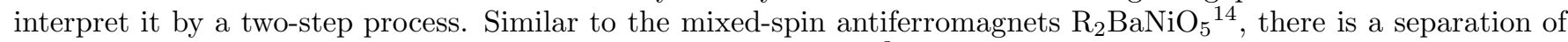
energy scales of magnetic excitations in $\mathrm{Cu}_{2} \mathrm{Fe}_{2} \mathrm{Ge}_{4} \mathrm{O}_{13}$. The $\mathrm{Fe}^{3+}$ centered magnons with low frequencies and the $\mathrm{Cu}^{2+}$ dimer centered triplons with high frequencies have different dynamical behaviors and different timescales. At the mean-field level, we first consider the effective Fe-Fe interaction $J_{\text {eff }}$ and the induced long range order in the $\mathrm{Fe}^{3+}$ sublattices. Goldstone modes appear at this stage. We then study the polarization of the $\mathrm{Cu}^{2+}$ dimers and its reaction on the $\mathrm{Fe}^{3+}$ sublattices. The hybridized magnons get a small gap due to the staggered field presented by the polarized $\mathrm{Cu}^{2+}$ dimers. This phenomenon has been studied earlier in spin- $\frac{1}{2}$ and spin- 1 antiferromagnetic spin chains experimentally $\stackrel{1,2,15}{\underline{1}}$ and theoretically ${ }^{16}$. It is shown that the presence of a staggered field will make the spin- $\frac{1}{2}$ antiferromagnetic chain gapful and split the Haldane triplet into two branches. In our studies, we further consider the fluctuations of the staggered field and their hybridization with the magnons. It may be interesting to consider the DM interaction or other anisotropic effects, which break the rotational symmetry and lead a gap naturally. We argue that the polarized $\mathrm{Cu}^{2+}$ dimers will make important contributions to the gap, even dominant if the anisotropies are tiny. We note that the estimated gap is close to the estimated $J_{\mathrm{DF}}$ from either the spin wave theor $\mathrm{y}^{\underline{6}}$ or the effective spin wave theory $\stackrel{4}{\text {. }}$.

In the case of $J_{\mathrm{DF}}<0$, the $\mathrm{Cu}^{2+}$ spin $\hat{T}_{n}$ will be parallel to its nearest $\mathrm{Fe}^{3+} \operatorname{spin} \hat{S}_{n}(n=1,2,3,4)$. Our theory is still applicable. The angle $\theta$ becomes $\theta^{\prime}=-\frac{1}{2} \arctan \frac{2\left|J_{\mathrm{DF}}\right| S}{J_{\mathrm{D}}}$. In Fig. 5, we also show the dependence of the energy gaps on $\frac{J_{\mathrm{DF}}}{J_{\mathrm{D}}}$ in the range of $J_{\mathrm{DF}}<0$. 


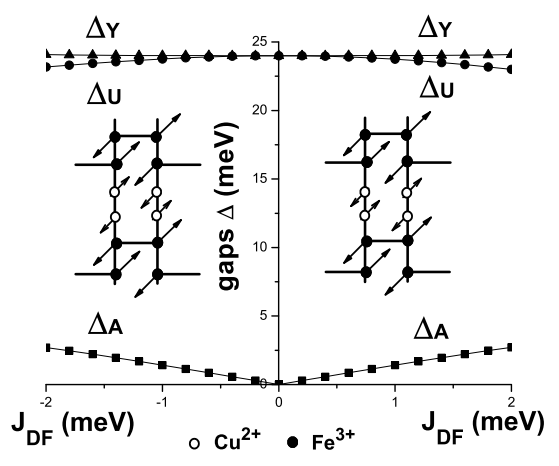

FIG. 5: The magnon-like excitations have a gap $\Delta_{A}$ proportional to $J_{\mathrm{DF}}$ (squares), while the triplon-like excitation shows $J_{\mathrm{DF}}^{4}$ $\left(\Delta_{Y}\right.$, triangles $)$ or $J_{\mathrm{DF}}^{2}$ dependences $\left(\Delta_{U}\right.$, circles $) . J_{\mathrm{D}}=24 \mathrm{meV}$ and $J_{\mathrm{a}}=1.6 \mathrm{meV}$. When $J_{\mathrm{DF}}<0$, the Cu${ }^{2+}$ spin is parallel to its nearest $\mathrm{Fe}^{3+}$ spin; while when $J_{\mathrm{DF}}>0$, they are antiparallel.

\section{SUMMARY}

In summary, we combine the bond-operator representation and Holstein-Primakoff transformation to study the mixed spin lattice model made up of $\mathrm{Cu}^{2+}\left(S=\frac{1}{2}\right)$ spin-dimers and $\mathrm{Fe}^{3+}\left(S=\frac{5}{2}\right)$ spin chains. A finite interaction $J_{\mathrm{DF}}$ between the spin-dimer and the spin chain introduces a cooperative order. The effective interaction between the spin chains, mediated by the spin dimer, is calculated up to the third order. It makes the $\mathrm{Fe}^{3+}$ spins ordered antiferromagnetically. Meanwhile, the local magnetization of $\mathrm{Fe}^{3+}$ spins polarizes the $\mathrm{Cu}^{2+}$ spin dimers and the staggered $\mathrm{Cu}^{2+}$ magnetization is shown proportional to $J_{\mathrm{DF}}$ as well as the local $\mathrm{Fe}^{3+}$ magnetization. It effectively presents a staggered field reacting on the $\mathrm{Fe}^{3+}$ spin chains. Considering the triplon-magnon interaction, the excitation spectra are especially investigated. The degeneracy of the triplons is lifted and the mode having longitudinal polarization and totally incompatible with conventional spin wave theory, as pointed out by Masuda et. al, is identified. It shows $\left(\frac{J_{\mathrm{DF}} S}{J_{\mathrm{D}}}\right)^{4}$ dependence near vanishing $J_{\mathrm{DF}}$. The hybridized triplon-like excitations get a weak dispersion and show a different $\left(\frac{J_{\mathrm{DF}}}{J_{\mathrm{D}}}\right)^{2}$ dependence. At the same time, the hybridized magnon-like excitations open a gap $\Delta_{A}=\Delta_{B} \approx J_{\mathrm{DF}} S \sqrt{\frac{2 J_{\mathrm{a}}}{J_{\mathrm{D}}}} \approx J_{\mathrm{DF}}$ at the momentum of $(0, \pi)$ or $(2 \pi, \pi)$. Away from this momentum, the magnon-like spectra show $\left(\frac{J_{\mathrm{DF}}}{J_{\mathrm{D}}}\right)^{2}$ relation, instead. The experiments on $\mathrm{Cu}_{2} \mathrm{Fe}_{2} \mathrm{Ge}_{4} \mathrm{O}_{13}$ are interpreted.

\section{ACKNOWLEDGEMENTS}

We acknowledge the financial support by Natural Science Foundation of China and 973-project under grant no. 2006CB921300 and 2006CB921400.

\section{APPENDIX A: EXCITATION SPECTRA}

From the matrix $H_{k}$ (eq. (14)), we get the equation to determine the excitation spectra:

$$
\omega^{8}+U \omega^{6}+V \omega^{4}+W \omega^{2}+Z=0,
$$

where, 


$$
\begin{aligned}
U= & -2\left(a^{2}+d^{2}-2 \delta^{2}+2 \gamma^{2} \cos \varphi\right) \\
V= & a^{4}+4 a^{2} d^{2}+d^{4}-4 a d \gamma^{2}+3 \gamma^{4}-4 a^{2} \delta^{2}-8 d^{2} \delta^{2}+2 \delta^{4}-2 \delta^{4} \cos k_{x}+4 \gamma^{2}\left(a^{2}+d^{2}-2 \delta^{2}\right) \cos \varphi \\
& +3 \gamma^{4} \cos ^{2} \varphi-4 d \gamma^{2} \delta \cos k_{y} \sin \varphi-3 \gamma^{4} \sin ^{2} \varphi \\
W= & -2 a^{4} d^{2}+4 a^{3} d \gamma^{2}+4 d^{4} \delta^{2}+2 \gamma^{4} \delta^{2}+4 a d \gamma^{2}\left(d^{2}-2 \delta^{2}\right)-a^{2}\left(2 d^{4}+\gamma^{4}-8 d^{2} \delta^{2}\right)-d^{2}\left(\gamma^{4}+4 \delta^{4}\right) \\
& +4 d \delta^{3} \cos k_{x}\left(d \delta+\gamma^{2} \cos k_{y} \sin \varphi\right)+\gamma^{2}\left(-\left(\gamma^{2}\left(a^{2}+d^{2}-2 \delta^{2}\right) \cos (2 \varphi)\right)-\gamma^{4} \cos (3 \varphi)\right. \\
& \left.+4 d \delta\left(a^{2}+d^{2}-\delta^{2}\right) \cos k_{y} \sin \varphi+\cos \varphi\left(-4 a^{2} d^{2}+8 a d \gamma^{2}-3 \gamma^{4}+8 d^{2} \delta^{2}+8 d \gamma^{2} \delta \cos k_{y} \sin \varphi\right)\right) \\
Z= & a^{4} d^{4}-4 a^{3} d^{3} \gamma^{2}+\frac{3 \gamma^{8}}{8}-\frac{5 d^{2} \gamma^{4} \delta^{2}}{2}+2 d^{4} \delta^{4}+a^{2}\left(5 d^{2} \gamma^{4}-4 d^{4} \delta^{2}\right)+2 a\left(-d \gamma^{6}+4 d^{3} \gamma^{2} \delta^{2}\right) \\
& -d^{2} \delta^{2} \cos k_{x}\left(\gamma^{4}+2 d^{2} \delta^{2}-\gamma^{4} \cos (2 \varphi)\right)+\frac{1}{8} \gamma^{4}\left(4\left(2 a^{2} d^{2}-4 a d \gamma^{2}+\gamma^{4}-3 d^{2} \delta^{2}\right) \cos (2 \varphi)+\gamma^{4} \cos (4 \varphi)\right) \\
& -2 d \gamma^{2} \delta \cos k_{y}\left(2 a^{2} d^{2}-4 a d \gamma^{2}+\gamma^{4}-2 d^{2} \delta^{2}+2 d^{2} \delta^{2} \cos k_{x}+\gamma^{4} \cos (2 \varphi)\right) \sin \varphi+d^{2} \gamma^{4} \delta^{2}\left(1+2 \cos \left(2 k_{y}\right)\right) \sin ^{2} \varphi
\end{aligned}
$$

with $\varphi=2\left(\theta+\frac{\pi}{4}\right)$.

Its roots are

$$
\omega_{\epsilon, \sigma}^{2}=-\frac{1}{4}\left(U+\epsilon \sqrt{8 \xi+U^{2}-4 V}\right)+\sigma \sqrt{\frac{1}{16}\left(U+\epsilon \sqrt{8 \xi+U^{2}-4 V}\right)^{2}-\left(\xi+\epsilon \frac{U \xi-W}{\sqrt{8 \xi+U^{2}-4 V}}\right)},
$$

where $\epsilon= \pm 1, \sigma= \pm 1$ and

$$
\xi=\frac{V}{6}+\left(-\frac{q}{2}+\sqrt{\left(\frac{q}{2}\right)^{2}+\left(\frac{p}{3}\right)^{3}}\right)^{1 / 3}+\left(-\frac{q}{2}-\sqrt{\left(\frac{q}{2}\right)^{2}+\left(\frac{p}{3}\right)^{3}}\right)^{1 / 3},
$$

with $p=\frac{U W}{4}-\frac{V^{2}}{12}-Z$ and $q=\frac{U V W}{24}-\frac{V^{3}}{108}-\frac{W^{2}}{8}-\frac{U^{2} Z}{8}+\frac{V Z}{3}$. If $\left|J_{\mathrm{DF}}\right| \ll J_{\mathrm{D}}$, the triplon-like excitation spectra can be expanded as

$$
\begin{aligned}
& \omega_{k}^{U_{\sigma}}=J_{\mathrm{D}}\left(1+g_{1}(k) \rho^{2}\right), \\
& \omega_{k}^{D_{\sigma}}=J_{\mathrm{D}}\left(1+g_{-1}(k) \rho^{2}\right), \quad \sigma=1,3,
\end{aligned}
$$

where, $\rho=-\frac{J_{\mathrm{DF}}}{J_{\mathrm{D}}} \sqrt{\frac{S}{2}}$ and

$$
g_{\epsilon}(k)=\frac{\eta\left(-4 \eta^{2}+8-\cos k_{x}\left(3 \eta^{2}-4+\eta^{2} \cos k_{y}\right)+2 \epsilon\left(\eta^{2}+\left(\eta^{2}-1\right) \cos k_{x}\right) \sqrt{3+\cos \left(2 k_{y}\right)}\right)}{-\eta^{4}+8 \eta^{2}-8+\eta^{4} \cos k_{y}}
$$

with $\eta=\frac{2 J_{\mathrm{a}} S}{J_{\mathrm{D}}}$. The energy gap of the triplon-like excitations

$$
\Delta_{U}=\Delta_{D} \approx J_{\mathrm{D}}\left(1-\frac{\eta}{1-\eta^{2}} \rho^{2}\right)
$$

locating at the momentum of $(2 \pi, \pi)$ (for the branch of $\left.\omega^{U_{\sigma}}, \sigma=1,3\right)$ or $(0, \pi)$ (for the branch of $\left.\omega^{D_{\sigma}}, \sigma=1,3\right)$. The band width of the triplon-like excitations is

$$
W_{U}=W_{D} \approx J_{\mathrm{D}} \frac{\eta}{1-\eta^{2}} \rho^{2}
$$

The energy gap of the magnon-like excitations occurs at the momentum of $(0, \pi)$ (for the branch of $\omega^{A_{\sigma}}, \sigma=1,3$ ) or $(2 \pi, \pi)$ (for the branch of $\left.\omega^{B_{\sigma}}, \sigma=2,4\right)$ and shows linear dependence on $J_{\mathrm{DF}}$ :

$$
\Delta_{A}=\Delta_{B}=J_{\mathrm{D}} \sqrt{\frac{2 \eta}{1-\eta^{2}}}|\rho| \approx\left|J_{\mathrm{DF}}\right| S \sqrt{\frac{2 J_{\mathrm{a}}}{J_{\mathrm{D}}}}|\approx| J_{\mathrm{DF}} \mid .
$$


Away from that momentum, the magnon-like excitation spectra can be expanded as

$$
\begin{aligned}
\omega_{k}^{A_{\sigma}} & \left.=J_{\mathrm{D}}\left[\sqrt{\frac{1}{2} \eta^{2}\left(1-\cos \frac{k_{x}}{2}\right.}\right)+g_{1}(k) \rho^{2}\right], \\
\omega_{k}^{B_{\sigma+1}} & =J_{\mathrm{D}}\left[\sqrt{\frac{1}{2} \eta^{2}\left(1+\cos \frac{k_{x}}{2}\right)}+g_{-1}(k) \rho^{2}\right], \quad \sigma=1,3 .
\end{aligned}
$$

Interestingly, they exhibit quadratic dependence on $\frac{J_{\mathrm{DF}}}{J_{\mathrm{D}}}$. The band width of the magnon-like excitations is

$$
W_{A}=W_{B} \approx J_{\mathrm{D}}\left(\eta-\sqrt{\frac{2 \eta}{1-\eta^{2}}}|\rho|\right) .
$$

1 A. Zheludev, J. P. Hill and D. J. Buttrey, Phys. Rev. B 54, 7216(1996).

2 A. Zheludev, E. Ressouche, S. Maslov, T. Yokoo, S. Raymond and J. Akimitsu, Phys. Rev. Lett. 80, 3630(1998).

3 T. Masuda, B. C. Chakoumakos, C. L. Nygren, S. Imai and K. Uchinokura, J. Solid State Chem. 176, 175(2003).

4 T. Masuda, A. Zheludev, B. Grenier, S. Imai, K. Uchinokura, E. Ressouche, and S. Park, Phys. Rev. Lett. 93, 077202(2004).

5 T. Masuda, A. Zheludev,B. Sales, S. Imai, K. Uchinokura, and S. Park, Phys. Rev.B 72, 094434(2005).

6 T. Masuda and K. Kakurai, M.Matsuda, K. Kaneko and N. Metoki, Phys. Rev.B 75, 220401(R)(2007).

7 M. Hase, M. Kohno, H. Kitazawa, O. Suzuki, K. Ozawa, G. Kido, M. Imai and X. Hu, Phys. Rev.B 72, 172412(2005).

8 T. Hamasaki, T. Ide, H. Kuroe, T. Sekine, M. Hase, I. Tsukada and T. Sakakibara, Phys. Rev.B 77, 134419(2008).

9 S. Sachdev and R. N. Bhatt, Phys. Rev. B 41, 9323(1990).

10 T. Holstein and H. Primakoff, Phys. Rev. 58, 1098(1940).

11 H.-T. Wang, Phys. Rev. B 65, 024426(2001).

12 M. Vojta and K. W. Becker, Phys. Rev. B 60, 15201(1999).

13 T. Sommer, M. Vojta and K. W. Becker, Eur. Phys. J. B 23, 329(2001).

14 A. Zheludev, S. Maslov, T. Yokoo, S. Raymond and J. Akimitsu, J. Phys.: Condens. Matter 13, R525(2001).

15 D. C. Dender, P. R. Hammar, D. H. Reich, C. Broholm and G. Appli, Phys. Rev. Lett. 79, 1750(1997).

16 M. Oshikawa and I. Affleck, Phys. Rev. Lett. 79, 2883(1997); S. Maslov and A. Zheludev, Phys. Rev. B 57, 68(1998); J. V. Alvarez, R. Valenti and A. Zheludev, Phys. Rev. B 65, 184417(2002); Jizhong Lou, Xi Dai, Shaojin Qin and Zhaobin Su, Phys. Rev. B 60, 52(1999); Jizhong Lou, Shaojin Qin, Changfeng Chen, Zhaobin Su and Lu Yu, Phys. Rev. B 65, 064420(2002) . 